\title{
Evaluation of MRI accuracy after primary systemic therapy in breast cancer patients considering tumor biology: optimizing the surgical planning
}

\author{
Alberto Bouzón ${ }^{1}$, Ángela Iglesias², Benigno Acea ${ }^{1}$, Cristina Mosquera ${ }^{1}$, Paz Santiago ${ }^{3}$, \\ Joaquín Mosquera²
}

${ }^{1}$ Department of Surgery, Breast Unit, Complejo Hospitalario Universitario A Coruña, Spain
${ }^{2}$ Department of Radiology, Breast Unit, Complejo Hospitalario Universitario A Coruña, Spain
${ }^{3}$ Department of Pathology, Breast Unit, Complejo Hospitalario Universitario A Coruña, Spain

Radiol Oncol 2019; 53(2): 171-177.

Received 25 December 2018

Accepted 18 April 2019

Correspondence to: Alberto Bouzón, M.D., Ph.D., Travesía Modesta Goicouría, №5, 16 izquierda, 15004, A Coruña, Spain. E-mail: dr.alberto@aecirujanos.es

Disclosure: No potential conflicts of interest were disclosed.

Background. We analyzed the accuracy of magnetic resonance imaging (MRI) after primary systemic therapy (PST) according to tumor subtype.

Patients and methods. Two-hundred and four breast cancer patients treated with PST were studied. MRI findings after PST were compared with pathologic findings, and results were stratified based on tumor subtype.

Results. Of the two-hundred and four breast cancer patients, eighty-four (41.2\%) achieved a pathologic complete response ( $\mathrm{PCR}$ ) in the breast. The MRI accuracy for predicting PCR was highest in triple-negative (TN) and HER2-positive (non-luminal) breast cancer (83.9 and $80.9 \%$, respectively). The mean size discrepancy between MRI-measured and pathologic residual tumor size was lowest in TN breast cancer and highest in luminal B-like (HER2-negative) breast cancer $(0.45 \mathrm{~cm}$ vs. $0.98 \mathrm{~cm}$, respectively; $P=0.003)$. After breast conserving surgery (BCS), we found a lower rate of positive margins in TN breast cancer and a higher rate of positive margins in luminal B-like (HER2-negative) breast cancer $(2.4 \%$ vs. $23.6 \%$, respectively).

Conclusions. If tumor response after PST is assessed by MRI, tumor subtype should be considered when BCS is planned. The accuracy of MRI is highest in TN breast cancer.

Key words: MRI; breast cancer; primary systemic therapy; tumor subtype

\section{Introduction}

Breast cancer is a heterogeneous disease stratified into several molecular subtypes with different behavior and prognosis. ${ }^{1,2}$ In clinical setting, breast cancer is routinely classified into approximated subtypes using immunohistochemistry according to hormone receptor (HR) and human epidermal growth factor 2 receptor (HER2) status.

Primary systemic therapy (PST) is the standard of care for locally advanced breast cancer, and it is increasingly being used for early breast cancer to improve cosmetic outcome after breast-conserving surgery (BCS). However, the primary goal of PST is to achieve pathologic complete response (pCR) prior to surgical treatment, which has been shown to predict favorable prognosis. ${ }^{3-5}$

Over the past few years, the highest use of PST was seen among HER2-positive and triplenegative breast cancer (TNBC) patients. ${ }^{6}$ Women with these tumor subtypes have the highest rates of BCS and pCR after PST. ${ }^{7}$ Furthermore, prognostic impact of pCR is highest in HER2-positive and TNBC. ${ }^{8-10}$ 
Currently, although the nuclear imaging techniques are promising, magnetic resonance imaging (MRI) is accepted as the most accurate imaging modality for assessment of tumor response and residual tumor size after PST in breast cancer patients. ${ }^{11}$ However, breast MRI is less effective for predicting $\mathrm{pCR} .{ }^{12-16}$ Therefore, in patients with absence of disease on MRI, surgical resection of the original tumor bed is required.

The purpose of the present study was to investigate the MRI diagnostic accuracy after PST in breast cancer patients focusing on tumor biology and its impact on margins after BCS. We also aimed to update the clinicopathologic factors affecting MRI accuracy to determine residual tumor size.

\section{Patients and methods}

\section{Patients}

A total of 204 patients with primary operable breast cancer treated with PST from October 2006 to September 2016 were included in this retrospective study. MRI was performed before and after PST to evaluate tumor response and residual tumor extent. A clip marker was placed at the tumor site prior to PST for surgical detection of the tumor bed. Patients with luminal A-like tumors were excluded due to their worse response to PST. Furthermore, in our previous study, the tumor size discrepancy between MRI and pathology was higher in this tumor subtype. ${ }^{16}$

\section{Subtype classification}

Breast cancer was classified into 5 approximate subtypes based on tumor characteristics using immunohistochemistry (HR status, HER2 status and ki-67 status). The five categories of tumor subtypes were: luminal A-like, luminal B-like (HER2negative), luminal B-like (HER2-positive), HER2 positive (non-luminal) and TN subtype. ${ }^{17}$ The cutoff of ki-67 expression level was established at 20\% to distinguish between luminal A-like and luminal B-like (HER2-negative) subtypes, so that a threshold of $\geq 20 \%$ was indicative of high ki-67 status.

\section{Chemotherapy regimen of PST}

$62.2 \%$ of patients received an anthracycline/taxane-based PST. All HER2-positive breast cancer patients except one received trastuzumab-based PST (36.3\%), in combination with an anthracycline/ taxane-based regimen. Three patients received a nanoparticle albumin-bound paclitaxel regimen $(1.5 \%)$.

\section{MRI protocol and assessment}

Residual tumor extent after PST was measured by MRI as the longest dimension of the enhancing lesion. MRI examinations were performed with patients in prone position using a $1.5 \mathrm{~T}$ MRI scanner (Best, The Netherlands) with breast-surface coils. The protocol included an axial T1-weighted sequence (repetition time [TR]: $494 \mathrm{msec}$, echo time [TE]: $8 \mathrm{msec}$, number of acquired signals: 2 , slice thickness: $3 \mathrm{~mm}$, interval: $0.03 \mathrm{~mm}$ ) and T2weighted sequence (TR: $5000 \mathrm{msec}, \mathrm{TE}: 120 \mathrm{msec}$, number of acquired signals: 2, slice thickness: 3 $\mathrm{mm}$, interval: $0.03 \mathrm{~mm}$ ), followed by diffusionweighted images performed at different $b$ values $(b$ $=0$ and $b=1000$ ).

A dynamic study (3D T1-weighted fast spoiled gradient-echo sequence) in the axial plane was performed before and 90, 180, 270, 360 and $450 \mathrm{sec}$ after starting intravenous injection of $0.1 \mathrm{mmol} / \mathrm{kg}$ of gadoterate meglumine (Gd-DOTA, DOTAREM, Guerbet) at a rate of $2 \mathrm{~mL} / \mathrm{s}$, followed by a $20 \mathrm{~mL}$ saline flush.

All images were processed at a workstation for the analysis of contrast enhancement, time-signal intensity curves and restriction to the difussion.

Imaging complete response (iCR) was defined as the absence of a clear enhancement visible on post-treatment MRI. Non-iCR was defined as the presence of any amount of tissue enhancement within the previous tumor bed visible on dynamic MRI after PST.

\section{Surgical management of breast tumors}

Surgery was performed within four weeks after completion of the neoadjuvant therapy. BCS was performed when the breast-tumor size index was favorable, considering patient's preference and multifocality. In non-palpable lesions after PST, a wire-guided resection of the clip containing breast area was performed. All patients undergoing BCS received adjuvant whole-breast irradiation with tangential fields.

\section{Pathological examination of surgical specimens}

All surgical specimens were fixed after gross evaluation in 10\% neutral-buffered formalin for 24 hours, and then serially cut into $5 \mathrm{~mm}$ thick sections. If 
residual tumor was clearly visible a gross measurement was made. If no evident tumor was identified, the clip marker placed prior to PST was found, and slides from the block containing the marker as well as the adjacent blocks were microscopically examined. Surgical specimens were stained with hematoxylin and eosin for the histological examination. The largest tumor diameter provided by the pathologist was used in the comparative study. If no invasive cancer was found in the surgical specimen after PST, regardless of the presence of carcinoma in situ, a breast pCR was considered. Negative resection margins were defined as no ink on tumor for invasive carcinoma and $2 \mathrm{~mm}$ clean margins for ductal carcinoma in situ.

\section{Statistical analysis}

Analyses were performed using SPSS version 23.0. Descriptive statistics of the variables included in the study were obtained. Continuous variables were expressed as mean (standard deviation) and median (range), and categorical variables were expressed as absolute values and percentages with their estimated $95 \%$ confidence interval. Student's $\mathrm{T}$ test or Mann-Whitney U Test were used to compare continuous variables. Moreover, chi-square test or Fisher's exact test were used to compare categorical variables. Multiple linear regression models were used to identify variables associated with MRI/pathologic tumor size discrepancy. Variables found to be significant on univariate analysis were included for the multivariate analysis. The diagnostic ability of MRI to detect residual disease after PST was quantified by the measures of diagnostic accuracy: sensitivity, specificity, positive predictive value (PPV), negative predictive value (NPV) and overall accuracy.

\section{Ethical issues}

Considering the Helsinki Declaration principles, the Institutional Research Ethics Committee approved this retrospective study (No. 2016/457).

\section{Results}

\section{Patient and tumor characteristics}

Baseline patient and tumor characteristics for the 204 breast cancer patients are described in Table 1. The median age of the patient cohort was 47 years (range, 30-82 years). Mean initial tumor size determined by MRI was $3.9 \mathrm{~cm}$ ( $72 \%$ of patients had T2
TABLE 1. Clinical and tumoral characteristics

\begin{tabular}{|c|c|c|c|c|}
\hline Variables & Mean & SD & Median & Range \\
\hline Age (years) & 49.4 & 11.6 & 47.0 & $30.0-82.0$ \\
\hline \multirow[t]{2}{*}{ Baseline tumor size $(\mathrm{cm})$} & 3.9 & 1.9 & 3.4 & $1.2-12.0$ \\
\hline & & $n$ & $\%$ & $95 \% \mathrm{Cl}$ \\
\hline \multirow{4}{*}{ Clinical tumor stage } & $\mathrm{Tl}$ & 21 & 10.3 & $5.9-14.7$ \\
\hline & T2 & 147 & 72.1 & $65.7-78.5$ \\
\hline & Т3 & 34 & 16.7 & $11.3-22.0$ \\
\hline & T4 & 2 & 0.9 & $0.1-3.5$ \\
\hline \multirow{2}{*}{ Histological type } & IDC & 196 & 96.1 & $93.2-99.0$ \\
\hline & ILC & 8 & 3.9 & $1.0-6.8$ \\
\hline \multirow{3}{*}{ Histological grade } & low-medium & 60 & 30.0 & $23.4-36.6$ \\
\hline & high & 140 & 70.0 & $63.4-76.6$ \\
\hline & NA & 4 & & \\
\hline \multirow{2}{*}{ Hormonal receptor status } & positive & 122 & 59.8 & $52.8-66.8$ \\
\hline & negative & 82 & 40.2 & $33.2-47.2$ \\
\hline \multirow{2}{*}{ HER2 status } & positive & 75 & 36.8 & $29.9-43.6$ \\
\hline & negative & 129 & 63.2 & $56.4-70.1$ \\
\hline \multirow{4}{*}{ Tumor subtype } & luminal B/HER2- & 77 & 37.7 & $30.8-44.6$ \\
\hline & luminal B/HER2+ & 45 & 22.1 & $16.1-28.0$ \\
\hline & HER2+ & 30 & 14.7 & $9.6-19.8$ \\
\hline & triple negative & 52 & 25.5 & 19.3-31.7 \\
\hline \multirow{2}{*}{ Baseline axillary status } & positive & 113 & 55.4 & $48.3-62.5$ \\
\hline & negative & 91 & 44.6 & $37.5-51.7$ \\
\hline
\end{tabular}

$\mathrm{Cl}=$ confidence interval; IDC = invasive ductal carcinoma; ILC = invasive lobular carcinoma: $\mathrm{NA}=$ not available; $\mathrm{SD}=$ standard deviation

tumors). Patients enrolled by tumor subtype were 77 luminal B-like (HER2-negative) (37.7\%), 45 luminal B-like (HER2-positive) (22.1\%), 30 HER2 positive (non-luminal) (14.7\%) and $52 \mathrm{TN}(25.5 \%)$. The axillary nodal status before PST was positive in $55.4 \%$ of cases.

\section{Response rate of the primary breast tumor to PST}

84 patients $(41.2 \%)$ achieved pCR in the breast after PST. The $\mathrm{pCR}$ rates differed significantly among tumor subtypes: $13 \%$ for luminal B-like (HER2negative), $42.2 \%$ for luminal B-like (HER2-positive), $76.6 \%$ for HER2 positive (non-luminal) and 61.5\% for TN. The iCR rate was $56.4 \%(115 / 204)$. The average pathologic tumor size was $1.10 \mathrm{~cm}$ and the average tumor size by post-treatment MRI was $1.03 \mathrm{~cm}$. 
TABLE 2. Diagnostic accuracy of MRI to detect residual disease

\begin{tabular}{lccccc}
\hline & S (\%) & SP (\%) & PPV (\%) & NPV (\%) & ACC (\%) \\
\hline Total & 62.5 & 83.3 & 84.3 & 60.9 & 71.1 \\
& & & & & \\
Luminal B/HER2- & 64.2 & 100.0 & 100.0 & 29.4 & 68.8 \\
Luminal B/HER2+ & 53.8 & 89.5 & 87.5 & 58.6 & 68.9 \\
HER2+ & 42.9 & 73.9 & 33.3 & 80.9 & 66.7 \\
Triple negative & 75.0 & 81.2 & 71.4 & 83.9 & 78.8 \\
\hline
\end{tabular}

ACC = accuracy; $N P V=$ negative predictive value; $P P V=$ positive predictive value; $S=$ sensitivity: $\mathrm{SP}=$ specificity

\section{Accuracy of MRI after PST}

The diagnostic accuracy of MRI to detect residual invasive disease in the breast for all patients and by tumor subtypes is summarized in Table 2 . The overall accuracy was $71.1 \%$. The NPV and PPV were $60.9 \%$ and $84.3 \%$, respectively. Among the different tumor subtypes, the highest diagnostic accuracy of MRI was observed in TNBC patients $(78.8 \%)$. The ability of MRI to predict pCR was highest for the TN and HER2 positive (non-luminal) subtypes $(83.9 \%$ and $80.9 \%$, respectively).

The mean size difference between post-treatment MRI and pathology was significantly lower in TN tumors as compared with luminal B-like (HER2-negative) tumors $(0.45 \mathrm{~cm}$ vs. 0.98; $\mathrm{p}=$ 0.003) (Figure 1).

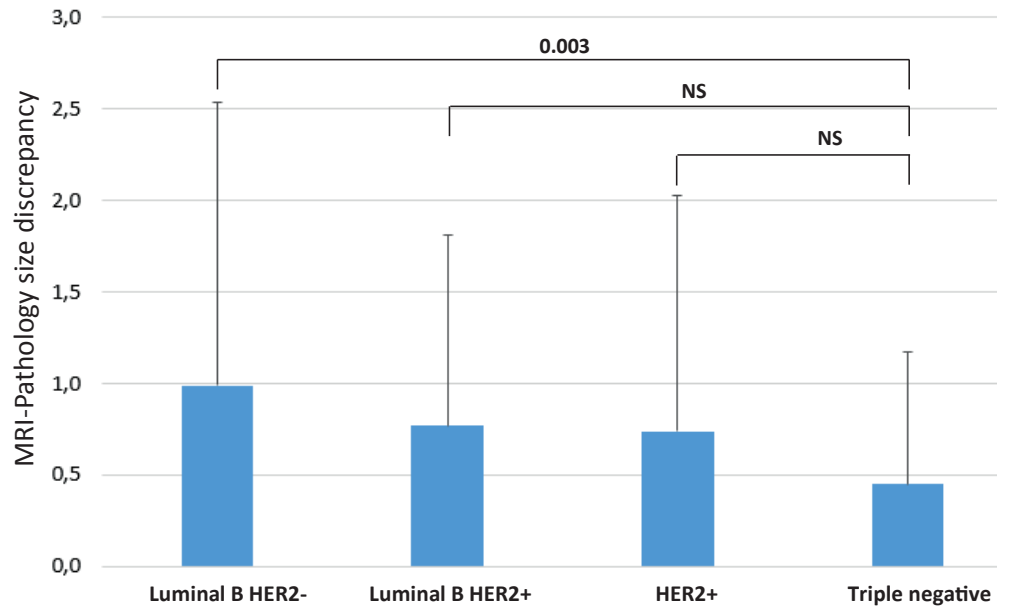

FIGURE 1. Diagnostic accuracy of MRI. Size discrepancy by tumor subtypes. Mean size discrepancy by tumor subtypes $(\mathrm{cm})$ : Iuminal B/HER2-: 0.98; luminal B/HER2+: 0.77; HER2+: 0.74; triple negative: 0.45 .
TABLE 3. Factors affecting the MRI accuracy based on the discrepancy between MRI and pathologic residual tumor size. Univariate and multivariate analysis

\begin{tabular}{|c|c|c|c|c|c|}
\hline \multicolumn{2}{|l|}{ Variable } & \multicolumn{3}{|c|}{$\begin{array}{l}\text { Mean size } \\
\text { discrepancy } \\
\quad( \pm \text { SD) }\end{array}$} & $p$ \\
\hline \multicolumn{2}{|l|}{$\begin{array}{l}\text { Age (years) } \\
\leq 45 \\
>45\end{array}$} & $\begin{array}{c}88 \\
115\end{array}$ & \multicolumn{2}{|c|}{$\begin{array}{l}0.72( \pm 0.95) \\
0.79( \pm 1.43)\end{array}$} & 0.668 \\
\hline \multicolumn{2}{|c|}{$\begin{array}{l}\text { Baseline fumor size }(\mathrm{cm}) \\
\leq 5 \\
>5\end{array}$} & $\begin{array}{c}167 \\
36\end{array}$ & \multicolumn{2}{|c|}{$\begin{array}{l}0.62( \pm 0.87) \\
1.39( \pm 2.18)\end{array}$} & 0.045 \\
\hline \multicolumn{2}{|c|}{$\begin{array}{l}\text { Histological type } \\
\text { IDC } \\
\text { ILC }\end{array}$} & $\begin{array}{c}195 \\
8\end{array}$ & \multicolumn{2}{|c|}{$\begin{array}{l}0.71( \pm 1.02) \\
2.05( \pm 3.75)\end{array}$} & 0.347 \\
\hline \multicolumn{2}{|l|}{$\begin{array}{l}\text { Tumor grade } \\
\text { Low-medium } \\
\text { high }\end{array}$} & $\begin{array}{c}60 \\
139\end{array}$ & \multicolumn{2}{|c|}{$\begin{array}{l}1.25( \pm 1.82) \\
0.55( \pm 0.82)\end{array}$} & 0.006 \\
\hline \multicolumn{2}{|c|}{$\begin{array}{l}\text { Hormonal receptor status } \\
\text { positive } \\
\text { negative }\end{array}$} & $\begin{array}{c}121 \\
82\end{array}$ & \multicolumn{2}{|c|}{$\begin{array}{l}0.90( \pm 1.38) \\
0.56( \pm 0.97)\end{array}$} & 0.059 \\
\hline \multicolumn{2}{|l|}{$\begin{array}{l}\text { HER2 status } \\
\text { positive } \\
\text { negative }\end{array}$} & $\begin{array}{c}74 \\
129\end{array}$ & \multicolumn{2}{|c|}{$\begin{array}{l}0.75( \pm 1.13) \\
0.76( \pm 1.30)\end{array}$} & 0.956 \\
\hline Variable & $\beta$ & se & $p$ & \multicolumn{2}{|c|}{$95 \% \mathrm{Cl}$} \\
\hline Tumor grade & 0.679 & 0.192 & 0.001 & 0.3 & -1.058 \\
\hline HR status & 0.214 & 0.181 & 0.239 & -0.1 & $3-0.570$ \\
\hline BTS (MRI) & 0.872 & 0.217 & $<0.001$ & 0.4 & -1.301 \\
\hline
\end{tabular}

${ }^{*} n$ (number of patients) $=203 ; \beta=$ regression coefficient; $\mathrm{BTS}=$ baseline tumor size; $\mathrm{Cl}=$ confidence interval; $\mathrm{HR}=$ hormonal receptor; $I D C=$ invasive ductal carcinoma; ILC = invasive lobular carcinoma; se = standard error; SD = standard desviation

Factors influencing the accuracy of MRI for predicting residual tumor size after PST

The mean discrepancy between residual tumor extent measured on MRI and pathology according to various clinicopathologic factors was determined in 203 patients (Table 3). Pathologic residual tumor size was not available in one case due to the presence of scattered residual multifocal disease in the surgical specimen. On univariable analysis, high tumor grade and baseline tumor size smaller than $5 \mathrm{~cm}$ were associated with a higher MRI accuracy. HR status showed marginal significance $(p=0.059)$, with lower mean discrepancy in HRnegative breast cancers. Age, histological type and HER2 status were not significantly associated with MRI/pathologic tumor size discrepancy. On multivariable analysis, tumor grade and baseline tumor size were significant and independent predictors of MRI accuracy. 


\section{Surgical treatment after PST}

After PST, 166 patients (81.4\%) underwent BCS and 38 patients $(18.6 \%)$ underwent mastectomy. From the BCS subset, 19 patients $(11.4 \%)$ required re-excision because of positive margins (3 of 19 patients required a salvage mastectomy). Of these 19 patients, $2(10.5 \%)$ had residual invasive disease on the final pathology. The positive margins rate after BCS was higher in patients with HR-positive breast cancer $(23.6 \%$ for luminal B-like (HER2-negative) and $13.5 \%$ for luminal B-like (HER2-positive)) than in patients with HER2 positive (non-luminal) or TNBC ( $5 \%$ and $2.4 \%$, respectively).

\section{Discussion}

PST is an increasing used treatment strategy for the management of operable breast cancer patients, whose main role in the surgical planning is to increase the rate of BCS.

Breast MRI is superior to conventional mammography and sonography for assessing tumor response after PST due to its ability to differentiate chemotherapy-induced fibrosis from residual disease. ${ }^{18-20}$

The accuracy of MRI for determining the presence and size of residual invasive disease should be considered when BCS is planned after PST. The underestimation of residual tumor size increases the rate of positive margins and the overestimation of residual tumor size may affect the cosmetic outcome if the tissue removal is very extensive. Our results demonstrate that the diagnostic accuracy of MRI is influenced by tumor subtype, being more effective in TNBC.

In the present study, the overall NPV of MRI was $60.9 \%$. However, MRI for predicting pCR is generally more accurate in those tumor subtypes with better response. In our study, MRI accurately predicted pCR in HER2 positive (non-luminal) and TNBC patients $(80.9 \%$ and $83.9 \%$, respectively) compared with HR-positive breast cancer patients. In a previous study, McGuire et al. ${ }^{14}$ found that the diagnostic accuracy of breast MRI for predicting pCR was higher in HR-negative breast cancer patients than in HR-positive breast cancer patients (73.6\% vs. $27.3 \%$, respectively). De Los Santos et al. ${ }^{13}$ concluded in their study that MRI accuracy differed significantly among breast cancer subtypes, and the highest NPV was observed in HER2 positive (nonluminal) and TNBC (62\% and 60\%, respectively).
Fukuda et al. ${ }^{15}$ reported that MRI was more effective for predicting pCR in TNBC (NPV $=72 \%)$.

The MRI accuracy for assessing tumor response depends on several factors, such as the used chemotherapeutic agents or the tumor regression pattern. ${ }^{21}$ PST in HER2-positive breast cancer patients includes targeted anti-HER2 agents and, therefore, the rate of achieving $\mathrm{pCR}$ is higher. In neoadjuvant setting, TNBC usually shows a concentric tumor regression pattern that allows MRI to be more accurate. $^{22}$

Our findings showed that baseline tumor size and tumor grade are significant and independent factors that affect MRI accuracy in predicting residual tumor extent after PST. In general, MRI accuracy is greater in tumors with better response to chemotherapy, such as high nuclear grade and cT1-2 breast cancers. ${ }^{23,24}$ Furthermore, MRI tend to be less accurate in HR-positive breast cancers. MRI may underestimate residual disease presenting as scattered cells within a large fibrotic region. ${ }^{25}$ This fragmented tumor regression pattern occurs more often in HR-positive breast cancers, leading to a higher rate of positive margins after BCS.

The present study showed that MRI was more accurate at predicting residual tumor size in TNBC patients, in whom the smallest MRI-pathology tumor size discrepancy (mean of $0.45 \mathrm{~cm}$ ) was observed. In addition, the rate of positive margins after BCS in TNBC patients was the lowest (2.4\%). The highest MRI-pathology tumor size discrepancy was observed in luminal B-like (HER2-negative) breast cancer patients (mean of $0.98 \mathrm{~cm}$ ) and, as a consequence, $23.6 \%$ of these women had positive margins after BCS. Previous studies also reported a higher MRI accuracy for predicting the residual tumor extent after PST in TNBC. ${ }^{26,27}$

These results indicate that it is necessary to consider tumor biology to optimize the planning of BCS based on MRI findings. When breast MRI describes a complete response in HER2 positive (nonluminal) and TN tumors, a minimal surgical resection of the tumor bed should be performed.

Novel breast imaging methods of evaluation of tumor response after PST are currently being studied. Contrast-enhanced spectral mammography is comparable to MRI in assessing residual disease after PST, but also has a limited accuracy to predict pCR. ${ }^{28}$ Stereotactic vaccum-assisted core needle biopsy of tumor bed after PST could help identify breast cancer patients with pCR to be included in prospective trials evaluating the safety of omitting surgical treatment. ${ }^{29}$ 
The strength of this study lies in the consideration of tumor subtypes in the analysis of MRI accuracy. Nonetheless, our study has several limitations. First, it is a retrospective study with a small number of HER2-positive (non-luminal) breast cancer patients $(n=30)$ compared to the number of patients presenting other tumor subtypes. During the study period, patients received different types of chemotherapy regimens, which may have affected the tumor response and, therefore, the MRI accuracy. Furthermore, the tumor response evaluation was performed using a 1.5-T MRI system, which seems to present lower diagnostic accuracy compared with 3-T MRI with a higher spatial resolution. ${ }^{30}$ Finally, only the absence of invasive residual disease was included in the definition of pCR in the breast.

In conclusion, if tumor response after PST is assessed by MRI, tumor subtype should be considered when BCS is planned. The accuracy of MRI is highest in TNBC patients.

\section{Acknowledgements}

The authors thank the team of the Department of Biostatistics of Complejo Hospitalario A Coruña for their contribution in the statistical analysis, specially Teresa Seoane.

\section{Abbreviations}

BCS = breast-conserving surgery; BTS = baseline tumor size; $\mathbf{H R}=$ hormone receptor; HER2 = human epidermal growth factor 2 receptor; $\mathbf{i C R}=$ imaging complete response; IDC = invasive ductal carcinoma; ILC invasive lobular carcinoma; $\mathbf{M R I}=$ magnetic resonance imaging; NPV = negative predictive value; $\mathbf{p C R}=$ pathologic complete response; $\mathbf{P P V}=$ positive predictive value; $\mathbf{P S T}=$ primary systemic therapy; $\mathbf{T N B C}=$ triple-negative breast cancer

\section{References}

1. Rouzier R, Perou CM, Symmans WF, Ibrahim N, Cristofanilli M, Anderson K, et al. Breast cancer molecular subtypes respond differently to preoperative chemotherapy. Clin Cancer Res 2005; 11: 5678-85. doi: 10.1158/1078-0432. CCR-04-2421

2. Yersal $\mathrm{O}$, Barutca S. Biological subtypes of breast cancer: prognostic and therapeutic implications. World J Clin Oncol 2014; 5: 412-24. doi: 10.5306/ wjco.v5.i3.412
3. Wolmark N, Wang J, Mamounas E, Bryant J, Fisher B. Preoperative chemotherapy in patients with operable breast cancer: nine-year results from National Surgical Adjuvant Breast and Bowel Project B-18. J Nat/ Cancer Inst Monogr 2001; 30: 96-102. PMID: 11773300

4. Gianni L, Baselga J, Eiermann W, Porta VG, Semiglazov V, Lluch A, et al. Phase III trial evaluating the addition of paclitaxel to doxorubicin followed by cyclophosphamide, methrotrexate, and fluorouracil, as adjuvant or primary systemic therapy: European Cooperative Trial in Operable Breast Cancer. $J$ Clin Oncol 2009; 27: 2474-81. doi: 10.1200/JCO. 2008.19.2567

5. Kong X, Moran MS, Zhang N, Haffty B, Yang Q. Meta-analysis confirms achieving pathological complete response after neoadjuvant chemotherapy predicts favourable prognosis for breast cancer patients. Eur J Cancer 2011; 47: 2084-90. doi: 10.1016/j.ejca.2011.06.014

6. Murphy BL, Day CN, Hoskin TL, Habermann EB, Boughey JC. Neoadjuvant chemotherapy use in breast cancer is greatest in excellent responsers: triple-negative and HER2+ subtypes. Ann Surg Oncol 2018; 25: 2241-8. doi: 10.1245/s10434-018-6531-5

7. Boughey J, McCall L, Ballman K, Mittendorf EA, Ahrendt GM, Wilke LG, et al. Tumor biology correlates with rates of breast-conserving surgery and pathologic complete response after neoadjuvant chemotherapy for breast cancer. Ann Surg 2014; 260: 608-16. doi: 10.1097/SLA. 0000000000000924

8. von Minckwitz G, Untch M, Blohmer JU, Costa SD, Eidtmann H, Fasching PA et al. Definition and impact of pathologic complete response on prognosis after neoadjuvant chemotherapy in various intrinsic breast cancer subtypes. J Clin Oncol 2012; 30: 1796-804. doi: 10.1200/JCO.2011. 38.8595

9. Cortazar P, Zhang L, Untch M, Mehta K, Costantino JP, Wolmark N, et al. Pathological complete response and long-term clinical benefit in breast cancer: the CTNeoBC pooled analysis. Lancet 2014; 384: 164-72. doi: 10.1016/ S0140-6736(13)62422-8

10. Boughey JC, Ballman KV, McCall LM, Mittendorf EA, Symmans WF, Julian TB, et al. Tumor biology and response to chemotherapy impact breast cancerspecific survival in node-positive breast cancer patients treated with neoadjuvant chemotherapy: long-term follow-up from ACOSOG Z1071 (Alliance). Ann Surg 2017; 266: 667-76. doi: 10.1097/SLA. 0000000000002373

11. Dialani V, Chadashvili T, Slanetz PJ. Role of imaging in neoadjuvant therapy for breast cancer. Ann Surg Oncol 2015; 22: 1416-24. doi: 10.1245/s10434015-4403-9

12. Straver ME, Loo CE, Rutgers EJ, Oldenburg HS, Wesseling J, Vrancken Peeters MJ, et al. MRI model to guide the surgical treatment in breast cancer patients after neoadjuvant chemotherapy. Ann Surg 2010; 251: 701-7. doi 10.1097/SLA. Ob013e3181c5dda3

13. De los Santos J, Cantor A, Amos KD, Forero A, Golshan M, Horton JK, et al. Magnetic resonance imaging as a predictor of pathologic response in patients treated with neoadjuvant systemic treatment for operable breast cancer. Translational Breast Cancer Research Consortium trial 017. Cancer 2013; 119: 1776-83. doi: 10.1002/cncr.27995

14. McGuire KP, Toro-Burguete J, Dang H, Young J, Soran A, Zuley M, et al. MRI staging after neoadjuvant chemotherapy for breast cancer: does tumor biology affect accuracy? Ann Surg Oncol 2011; 18: 3149-54. doi: 10.1245/ s10434-011-1912-z

15. Fukuda T, Horii R, Gomi N, Miyagi Y, Takahashi S, Ito Y, et al. Accuracy of magnetic resonance imaging for predicting pathological complete response of breast cancer after neoadjuvant chemotherapy: association with breast cancer subtype. Springerplus 2016; 5: 152. doi: 10.1186/s40064-016-1800-x

16. Bouzón A, Acea B, Soler R, Iglesias Á, Santiago P, Mosquera J, et al Diagnostic accuracy of MRI to evaluate tomour response and residual tumour size after neoadjuvant chemotherapy in breast cancer patients. Radiol Oncol 2016; 50: 73-9. doi: 10.1515/raon-2016-0007

17. Goldhirsch A, Winer EP, Coates AS, Gelber RD, Piccart-Gebhart M, Thürlimann B, et al. Personalizing the treatment of women with early breast cancer: highlights of the St Gallen International Expert Consensus on the Primary Therapy of Early Breast Cancer 2013. Ann Oncol 2013; 24: 2206-23. doi: 10.1093/annonc/mdt303

18. Rosen EL, Blackwell KL, Baker JA, Soo MS, Bentley RC, Yu D, et al. Accuracy of $\mathrm{MRI}$ in the detection of residual breast cancer after neoadjuvant chemotherapy. Am J Roentgenol 2003; 181: 1275-82. doi: 10.2214/ ajr.181.5.1811275 
19. Yeh E, Slanetz P, Kopans DB, Rafferty E, Georgian-Smith D, Moy L, et al. Prospective comparison of mammography, sonography, and MRI in patients undergoing neoadjuvant chemotherapy for palpable breast cancer. Am J Roentgenol 2005; 184: 868-77. doi: 10.2214/ajr.184.3.01840868

20. Croshaw R, Shapiro-Wright H, Svensson E, Erb K, Julian T. Accuracy of clinical examination, digital mammogram, ultrasound, and MRI in determining postneoadjuvant pathologic tumor response in operable breast cancer patients. Ann Surg Oncol 2011; 18: 3160-3. doi: 10.1245/s10434-011-1919-5

21. Orel $\mathrm{S}$. Who should have breast magnetic resonance imaging evaluation? J Clin Oncol 2008; 26: 703-11. doi: 10.1200/JCO.2007.14.3594

22. Eom HJ, Cha JH, Choi WJ, Chae EY, Shin HJ, Kim HH. Predictive clinicopathologic and dynamic contrast-enhanced MRI findings for tumor response to neoadjuvant chemotherapy in triple-negative breast cancer. Am J Roentgenol 2017; 208: 225-30. doi: 10.2214/AJR.16.17125

23. Wang J, Buchholz TA, Middleton L, Allred DC, Tucker SL, Kuerer HM, et al. Assessment of histologic features and expression of biomarkers in predicting pathologic response to anthracycline-based neoadjuvant chemotherapy in patients with breast cancer. Cancer 2002; 94: 3107-14. doi: 10.1002/ cncr.10585

24. Goorts B, van Nijnatten TJ, de Munck L, Moossdorff M, Heuts EM, de Boer $\mathrm{M}$, et al. Clinical tumor stage is the most important predictor of pathological complete response rate after neoadjuvant chemotherapy in breast cancer patients. Breast Cancer Res Treat 2017; 163: 83-91. doi: 10.1007/s10549017-4155-2

25. Bahri S, Chen JH, Mehta RS, Carpenter PM, Nie K, Kwon SY, et al. Residual breast cancer diagnosed by MRI in patients receiving neoadjuvant chemotherapy with and without bevacizumab. Ann Surg Oncol 2009; 16: 1619-28. doi: 10.1245/s10434-009-0441-5

26. Ko ES, Han BK, Kim RB, Ko EY, Shin JH, Hahn SY, et al. Analysis of factors that influence the accuracy of magnetic resonance imaging for predicting response after neoadjuvant chemotherapy in locally advanced breast cancer. Ann Surg Oncol 2013; 20: 2562-8. doi: 10.1245/s10434-013-2925-6

27. Moon H-G, Han W, Ahn SK, Cho N, Moon WK, Im SA, et al. Breast cancer molecular phenotype and the use of HER2-targeted agents influence the accuracy of breast MRI after neoadjuvant chemotherapy. Ann Surg 2013; 257: 133-7. doi:10.1097/SLA.0b013e3182686bd9

28. Patel BK, Hilal T, Covington M, Zhang $\mathrm{N}$, Kosiorek HE, Lobbes $\mathrm{M}$, et al. Contrast-enhanced spectral mammography is comparable to MRI in the assessement of residual breast cancer following neoadjuvant systemic therapy. Ann Surg Oncol 2018; 25: 1350-6. doi: 10.1245/ s10434-018-6413-x

29. Rauch GM, Kuerer HM, Adrada B, Santiago L, Moseley T, Candelaria RP, et al. Biopsy feasibility trial for breast cancer pathologic complete response detection after neoadjuvant chemotherapy: imaging assessment and correlation endpoints. Ann Surg Oncol 2018; 25: 1953-60. doi:10.1245/ s10434-018-6481-y

30. Heldahl MG, Lundgren S, Jensen LR, Gribbestad IS, Bathen TF. Monitoring neoadjuvant chemotherapy in breast cancer patients: improved MR assessment at 3 T? J Magn Reson Imaging 2011; 34: 547-56. doi: 10.1002/ jmri.22642 